\title{
Multi-modal SEM/FIB-SEM for Precise Targeting of Cell-Cell Junctions in Human Pancreatic Islets.
}

Jing W. Hughes ${ }^{1}$, David W. Piston ${ }^{1}$, Matthew S. Joens ${ }^{2}$ and James A.J. Fitzpatrick ${ }^{2}$

1. Washington University School of Medicine, Department of Cell Biology, Saint Louis, USA

2. Washington University Center for Cellular Imaging, Washington University School of Medicine, Saint Louis, USA

Although much research has been done to generate EM-resolution 3D models of rodent islets [1,2], the use of human islets has been relatively unexplored. To analyze these, we developed a pipeline to rapidly and precisely screen fixed and stained human islets for high-resolution focused ion beam (FIB) nanotomography. Upon close examination, we find physical evidence of granule exchange between islet endocrine cells, as well as junctional structures previous not described in islets. The novelty of this work is two-fold: the application of FIB-SEM to studying human islet tissue, and the discovery of new structural elements that can potentially link morphological and functional aspects of islet anatomy.

Healthy human donor islets were obtained through the NIH-funded Integrated Islet Distribution Program (iidp.coh.org). Using methods we have developed for large-area acquisitions [3], we were able to take a single 70nm slice, place it directly onto a silicon wafer chip, and image all exposed islets on the blockface in the SEM at high-speed and high-resolution $(10 \mathrm{~nm} /$ pixel $)$ using a solid-state backscatter detector (Fig. 1). By imaging an adjacent thin section as opposed to the blockface itself, we reduce the risk of any negative beam-blockface interactions when imaging with a higher accelerating voltage (8 $\mathrm{KeV})$ necessary to produce a strong backscatter signal with high signal to noise at fast scan speeds ( $2 \mu \mathrm{s}$ pixel dwell, line average 3). With this "cellular-level map" we were then able to precisely correlate regions of interest that fell upon specific boundaries such as alpha/beta or beta/delta cell junctions or capillary borders, and set up a FIB nanotomography acquisition in this precise location. Using the ATLAS engine (Fibics, Ottawa, Canada) for three-dimensional nanotomography data acquisition, we were able to acquire data at $12 \mathrm{~nm} /$ pixel with $12 \mathrm{~nm}$ slices. The volumes acquired were approximately $30 \mu \mathrm{m} \times 30 \mu \mathrm{m} \times 25 \mu \mathrm{m}$ and each slice was acquired in 40-60 seconds with simultaneous imaging/milling for a total of 40-48 hours of imaging per region of interest.

The ability to acquire isometric voxels in the FIB compared to other methodologies [4] such as serial blockface imaging or array tomography allows for a much easier and uncompromised visualization of orthogonal image planes, making identification of junction complexes and desmosomes faster and more robust. Although the acquired 3D volume in the FIB is significantly smaller than can be obtained using its counterpart methods, the ability to combine large area arrays with high resolution FIB nanotomography allows both quantitative and qualitative analysis of cellular morphology for both diabetic and healthy human islets.

Using these methods, we capture for the first time the passage of a secretory granule in transit from a beta cell to a neighboring alpha cell (Figure 1D, granule in green). Insulin granule passage has been reported between beta cells and resident islet macrophages [5], and because insulin is a major diabetes autoantigen, this is thought to represent a triggering event for autoimmune activation in type 1 diabetes. The physiologic implications of granule transfer among endocrine cells are less clear, nor do we yet understand whether this is a constitutive or regulated process. The sharing of secretory granules may 
contribute to the creation or maintenance of the insulin/glucagon $(\beta / \alpha)$ bi-hormonal islet cell [6]. In addition to granule passage, we observed other areas of intense membrane activity between different islet cells, as well as classic adhesion structures including desmosomes and junctional complexes (Figure 2). Desmosomal structures have been observed by EM in human pancreas but only at the junction between acinar and islet tissue [7]. The discovery of desmosomes within the endocrine islet is novel and suggests a role in cell adhesion, possibly to preserve islet structural integrity and to enhance paracrine signaling among alpha, beta, and delta cells. Future work includes comparing non-diabetic and diabetic human pancreata to detect changes in intra-islet cellular interactions that occur with disease [8].

\section{References:}

[1] A Shomorony et al, J. Microsc. 259 (2015), p. 155.

[2] CR Pfeifer et al, J. Struct. Biol. 189 (2015), p. 44.

[3] BE Kerman et al, Development 142 (2015), p. 2213.

[4] CJ Peddie and LM Collinson, Micron 61 (2014), p. 9.

[5] AN Vomund et al, Proc. Natl. Acad. Sci. USA 112 (2015), p. 5496.

[6] T Mezza et al, J. Clin. Endocrinol. Metab. 101 (2016), p. 470.

[7] K Aida et al, PLOS ONE 9 (2014) p. e95110.

[8] The authors acknowledge funding from the Washington University Center for Cellular Imaging micro-grant program.
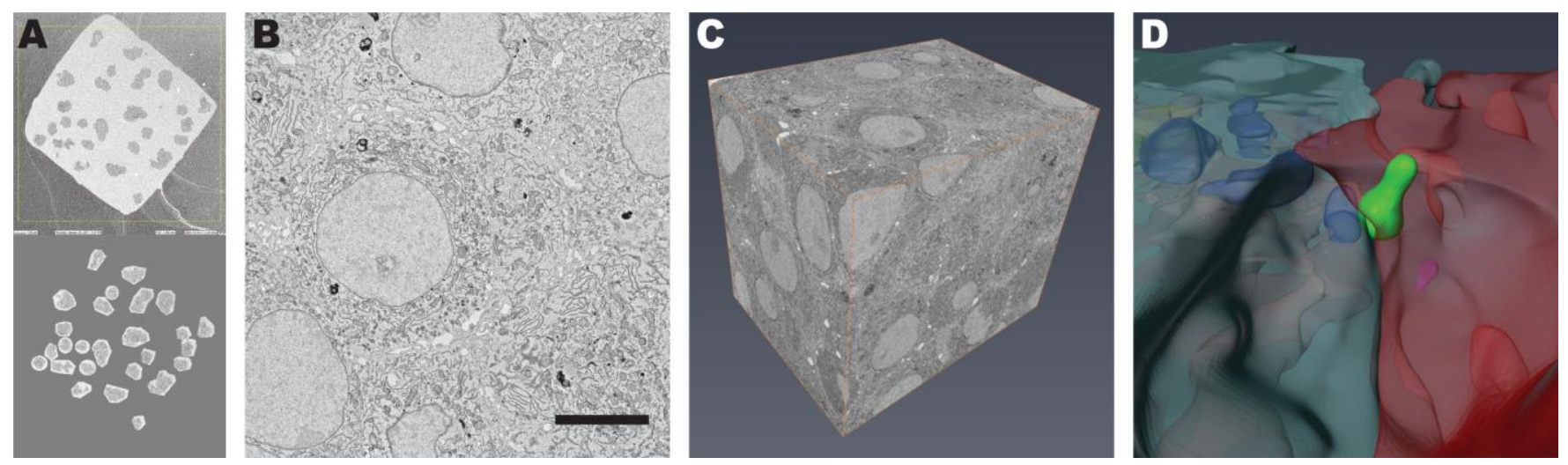

Figure 1. Workflow for targeted FIB analysis of human islets. A) A single 70nm slice is placed on a wafer chip and islets are imaged via SEM. B) A region of interest is selected based off of cell borders. C) $3 \mathrm{D}$ block is acquired with $12 \mathrm{~nm}$ isometric voxels at high speed. D) A subvolume is segmented to show insulin granule transport (green) between a beta cell (blue) and an alpha cell (red). Scale: $5 \mu \mathrm{m}$.
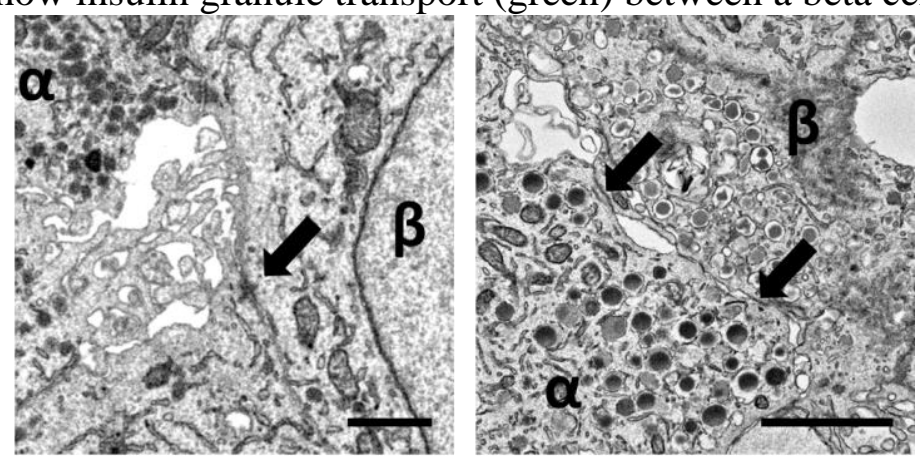

Figure 2. Cell adhesion structures between human islet endocrine cells. A) Desmosome (arrow) between an alpha and beta cell. B) Junctional complexes between an alpha and beta cell. Scale: $1 \mu \mathrm{m}$. 